\title{
Phronesis
}

\section{Formation à distance et activité du stagiaire de la formation professionnelle continue}

\section{La lettre de mission comme outil d'ajustement et de régulation Distance learning and activity of the trainee of continuing vocational training The engagement letter as a tool for adjustment and control}

\section{Béatrice Verquin-Savarieau}

Volume 3, numéro 1-2, janvier-avril 2014

Le stage en formation, tendances et résistances

URI : https://id.erudit.org/iderudit/1024588ar

DOI : https://doi.org/10.7202/1024588ar

Aller au sommaire du numéro

\section{Éditeur(s)}

Université de Sherbrooke

ISSN

1925-4873 (numérique)

Découvrir la revue

Citer cet article

Verquin-Savarieau, B. (2014). Formation à distance et activité du stagiaire de la formation professionnelle continue : la lettre de mission comme outil

d'ajustement et de régulation. Phronesis, 3(1-2), 49-57.

https://doi.org/10.7202/1024588ar

\section{Résumé de l'article}

Quels contenus des lettres de missions pour quelles démarches et activités d'ingénierie de formation, pour des étudiants salariés en activité et suivant leurs études à distance ? Cet article questionne le stage de formation professionnelle continue dans le cadre d'une formation hybride. Il s'agit d'interroger en particulier la conception de la mission de stage, en tant que contenu opératoire facilitant les transformations des sujets dans l'action. Nous présenterons le rôle crucial joué par la lettre de mission, en tant qu'outil d'ajustement et de régulation d'une commande ou d'une auto-commande aux attentes de l'université. La méthodologie présentée consiste dans une grille d'analyse des démarches d'ingénierie de formation, construite à partir de la lecture de quatre domaines d'activités. L'analyse des activités n'est pas dans ce dispositif une modalité de formation, mais nous permet de questionner la conception du dispositif de formation. 


\title{
Formation à distance et activité du stagiaire de la formation professionnelle continue La lettre de mission comme outil d'ajustement et de régulation
}

\section{CIVIIC}

\author{
Béatrice VERQUIN-SAVARIEAU
}

Université de Rouen

1 Rue Thomas Becket, 76821 Mont-Saint-Aignan

Beatrice.Savarieau@univ-rouen.fr.

Mots-clés : Dispositif hybride, expérience, activité, stage, professionnalité, ingénierie de formation, lettre de mission

Résumé : Quels contenus des lettres de missions pour quelles démarches et activités d’ingénierie de formation, pour des étudiants salariés en activité et suivant leurs études à distance? Cet article questionne le stage de formation professionnelle continue dans le cadre d'une formation hybride. Il s'agit d'interroger en particulier la conception de la mission de stage, en tant que contenu opératoire facilitant les transformations des sujets dans l'action. Nous présenterons le rôle crucial joué par la lettre de mission, en tant qu'outil d'ajustement et de régulation d'une commande ou d'une auto-commande aux attentes de l'université. La méthodologie présentée consiste dans une grille d'analyse des démarches d'ingénierie de formation, construite à partir de la lecture de quatre domaines d'activités. L'analyse des activités n'est pas dans ce dispositif une modalité de formation, mais nous permet de questionner la conception du dispositif de formation.

Title : Distance learning and activity of the trainee of continuing vocational training. The engagement letter as a tool for adjustment and control

Key-words : Hybrid device, experience, activity, vocational education training professionality, engineering of training

Abstract : This paper discusses the practical vocational training in the framework of a hybrid system. This is particularly ask the vocationnal education training as operational content to facilitate the transformation of subjects in action. We present the crucial role played by the engagement letter, as a tool for adjustment and regulation to the expectations of the university. The methodology presented is in analytical engineering training approaches, built from reading four business areas. Analysis of the activities is not a device in this type of training, but allows us to question the design of the training. 


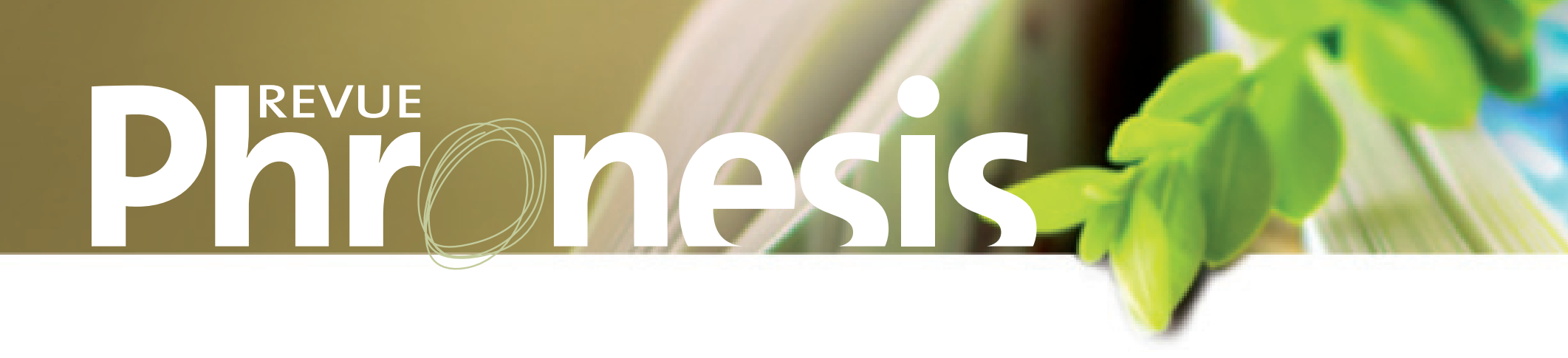

\section{Introduction}

Cette recherche questionne le stage de formation professionnelle continue dans le cadre d'une formation hybride du campus numérique Forse, soit le master 2 professionnel « Métiers de la formation» de l'université de Rouen. Ces étudiants apprennent au travail, (Bourgeois et Durand, 2012 ; Depover et Marchand, 2002) et cette situation nest pas sans interroger les démarches et activités réalisées au titre de leur stage, mais aussi le manque de distanciation que l'on observe entre les lieux de la réflexion et de l'action. Il s'agira par conséquent d’élaborer un contenu et un suivi de stage, prenant en compte un processus de territorialisation/déterritorialisation, au sens d'un espace social vécu en construction, (Paquelin et al., 2006) ; soit d’aborder la formation comme un «territoire ", que Di Meo (2001) définit comme étant «l'espace de l'action et l'objet d'une appropriation ou réappropriation sociale des conditions de l'action».

Le dispositif de formation et son environnement interrogent le processus d'apprentissage. "La formation expérientielle soppose à la formation institutionnelle » (Houssaye, 2012), dans le sens où elle brouille les repères pédagogiques habituels et oblige à repenser la place, le but et les modalités de l'expérience au sein de ce dispositif. Elle ne constitue pas alors ce que Bourgeois et Nizet (1997) appellent un " espace protégé ». Nous interrogerons de ce fait l'expérience du stagiaire qui n’est pas un vécu que l’on peut quantifier en termes de durée, mais le travail qui seffectue sur le vécu, y compris en prenant en compte le parcours professionnel antérieur (Pastré, 2013). Les activités réalisées nécessitent alors dêtre accompagnées dans un va et vient permanent, afin de pouvoir distinguer ce qui relève de la mission de stage, de l'activité professionnelle ou de la recherche action qui donnera lieu à l'écriture d'un mémoire. "Théorie » et "pratique " ou " réflexion " et « action", à l'instar de ce que préconisait Dewey (1938) sont ici deux fonctions fondamentales de l'activité du sujet, en transformation cognitive et transaction continue avec son environnement par l'action, mais pour cela chaque étudiant doit concevoir ses propres repères et organisations de travail.

Quels contenus des lettres de missions pour quelles démarches et activités d'ingénierie de formation, pour des étudiants salariés en activité et suivant leurs études à distance ? Nous interrogerons la conception de la mission de stage, en tant que contenu opératoire facilitant les transformations des sujets dans l'action. Nous présenterons tout d’abord notre cadre théorique construit autour de : l'activité (Albarello, Barbier, Bourgeois et Durand, 2013 ; Champy-Remoussenard, 2005 ; Leplat, 1997), l'expérience (Madelrieux, 2010 ; Mayen, 2009), et de la professionnalité (Aballéa, 1992 ; Perez-Roux, 2012). Seront ensuite abordées, les caractéristiques du dispositif hybride du diplôme considéré et la méthodologie de la recherche, basée sur une grille d'analyse des lettres de missions de stages. Nous finirons enfin par la présentation de nos résultats et de quelques perspectives autour de notre objet de recherche.

\section{Conception des missions de stage et vécu de l'activité}

\subsection{Le vécu de l'activité et ses conditions de mise en ouvre}

La conception d'une mission de stage, basée sur la déclaration d'activités à mettre en œuvre, qui feront l'objet d'une évaluation, questionne tout autant le contexte de la formation que les contraintes qui en résultent. Si un dispositif hybride favorise la proximité de l’apprentissage possible en contexte réel et la disponibilité des ressources éducatives (Depover et Marchand, 2002), il comporte son lot de contraintes, notamment les chevauchements rendus possibles entre : espace public et espace privé, temps du travail et hors temps de travail, identité professionnelle et étudiante ; chevauchements qui impliquent des conflits et des tiraillements qu'il est nécessaire de résoudre. Cet inconfort du dedans/ dehors nécessite que la mission soit explicite et comprise par tous, que ce soit dans le lieu de travail ou dans celui de la formation. Cette mission de stage (point de vue institutionnel) très enracinée dans l'espace professionnel (formation expérientielle) pose également la question de la coopération et de la compréhension des collègues face aux transformations du sujet recherchées. Elle interroge aussi les temporalités de l'apprentissage, soit le temps nécessaire pour pouvoir repenser ses habitudes, au sens défini par Dewey (2006), d' " habitude d'activité ", soit l'apprentissage perçu comme une manière d'agir caractéristique d'un sujet dans une situation récurrente, investie et transformée dans l'exercice même de l'activité. L'activité n’est donc pas uniquement une construction, elle est aussi reconstruction, elle peut même devenir une reconstruction continue.

Pour Leplat (2000), l'activité est une réponse du sujet aux exigences d'une tâche, elle comporte une part d’adaptation aux conditions de l'action. Ainsi, toute activité humaine est guidée par deux paramètres, l'intention ou le plan et les caractéristiques de l'environnement, soit dans un rapport dynamique de codétermination qui s'influencent réciproquement et contribuent à leur mutuelle transformation. Pour 
Barbier (2013), il est indispensable de distinguer l’activité des activités. L'activité étant un ensemble de processus dans lesquels et par lesquels un acteur social (individu ou groupe) peut être impliqué et transformé, dans ses rapports avec ses environnements physiques, sociaux et/ou mentaux. L'activité rejoignant le vécu, ce dernier n’est pas dissociable de l'activité. Cette activité interroge également la posture de l'apprenant, dans son rapport à lui-même, aux autres et au réel, notamment en tant que praticien en voie de transformation, dans son projet de formation, que nous pourrions résumer comme étant "l'anticipation d’un individu perfectible " (Boutinet, 1990). Lactivité questionne également l'identité professionnelle présente et à venir, impactant de ce fait le choix d'une posture, qu'il affirme ou qu'il donne à voir. La posture du stagiaire sera à analyser, au sens décrit par Ardoino (1990) comme étant une "position ", soit un système d'attitudes et de regards vis-à-vis des partenaires, des situations, des objets, manifestant en cela des représentations, des intentionnalités ou des stratégies.

\subsection{De l'expérience à l'expérience vécue de l'activité}

C'est ainsi qu'un certain nombre d'auteurs parviennent à décrire les processus d'apprentissage chez l'adulte (Albarello, Barbier, Bourgeois et Durand, 2013 ; Bourgeois et Nizet, 1997) comme un mode de création de savoirs à partir de la transformation de l'expérience soit un mouvement de rétroaction selon le cycle d'apprentissage de Kolb (1984), qui amène à reconsidérer une expérience au travers d'une observation réfléchie et attentive de la situation, puis d'entrer dans la conceptualisation de l'action qui s’appuie sur des mécanismes de prise de conscience, afin de parvenir à reconstruire par la distanciation de son activité, son action à un autre niveau. Lexpérience humaine est une et se poursuit d'une activité d’apprentissage à une autre. Ainsi, les professionnels en formation s'appuient sur l'expérience du travail qu'ils ont acquise, ils parlent beaucoup plus facilement en praticiens et cette expérience peut parfois, comme l’affirme Pastré (2013, p. 97), les enfermer « dans la complexité d'une pratique, mais aussi dans la singularité d'une situation ». L’expérience que nous questionnons dans cette recherche s'apparente à celle qui consiste à «faire l'expérience de », soit celle que l'on désigne couramment sous le terme " d'expérience vécue », comme confrontation à une situation, ou pratique, ou conception, ou représentation nouvelle; de laquelle s'en suit un ajustement des sujets dans les interactions avec les personnes ou les objets qui les entourent. L'expérience vécue constitue donc lensemble des opérations mentales et actionnelles qui permettent la transformation de ressources pour l'activité de la personne, y compris pour les activités futures (Balleux, 2000).

\subsection{Que faut-il entendre par professionnalité?}

Le sujet élabore alors son expérience à partir de l'activité qu'il a réalisée. Il renforce ainsi sa professionnalité, soit « l’ensemble des compétences professionnelles mobilisées dans l'exercice d'une profession, sous le double point de vue de l'activité et de l'identité " (Perez-Roux, 2012, p. 11). L'expérience s'inscrit alors dans ce processus de transformation de lêtre, dans ce sens quelle peut être définie comme étant ce que je fais de ce qui m’advient, (Barbier 2013, p. 73). S’en suit alors que la professionnalité est perçue comme étant un ensemble de compétences, capacités, savoirs, connaissances et identités reconnus par une organisation ou un groupe professionnel, soit comme étant les caractéristiques d'un professionnel reconnu par ses pairs pour son professionnalisme. Nous ne parlons pas de "professionnel compétent ", "référence mythique » selon Caspar (2011, p. 98) et qui manifeste la démarche d'ingénierie des compétences, plutôt que celle déducation à laquelle nous préférons nous référer.

La professionnalité suppose donc la construction ou la transformation d'une identité professionnelle, à partir d'un vécu et l'acquisition d'un corpus de connaissances et de compétences contribuant à cette reconnaissance. Toute construction de cette dernière ne peut relever d'une démarche individuelle, elle s'insère dans une dynamique sociale et institutionnelle, soit un processus de socialisation du travail. Pour Aballéa (1992, p. 46), la professionnalité est l'un des attributs d'un individu ou d'un groupe qui a su développer « une expertise complexe et composite, encadrée par un système de références, valeurs et normes, de mises en ouvre », ou " un savoir et une déontologie, sinon une science et une conscience ». La socialisation résulte alors des interactions au sein d'un double mouvement : de montée en compétences et en qualification d'une part ; et de la reconnaissance de cette professionnalité par les pairs et l'environnement d'autre part. Cette professionnalité, en tant que compétences, se doit dêtre mise en acte pour donner toute sa visibilité et permettre la reconnaissance.

\section{Le contexte de la recherche : un dispositif professionnalisant hybride}

\subsection{Les objectifs du diplôme}

Lobjectif de ce master est de former des «spécialistes polyvalents » qui accompagneront de façon pluridimensionnelle l'aménagement et le développement de projets de formation dans le champ des politiques territoriales, des ressources humaines ou de la médiation éducative. Il vise notamment à permettre à létudiant de se construire un socle de savoirs (connaissances et pratiques) dans le champ de la formation ; à développer un savoir-faire et une culture de lévaluation ; à former aux démarches de l'ingénierie de formation (analyse, conception, mise en place, conduite et développement de systèmes de formation); à produire des compétences professionnelles nouvelles mises en ouvre dans le cadre du stage. L'ingénierie de formation est enseignée comme étant : " Une démarche socioprofessionnelle où l'ingénieur-formation a par des méthodologies appropriées, à analyser, concevoir, réaliser et évaluer des actions, dispositifs et/ou systèmes de formation en tenant compte de l'environnement et des acteurs professionnels » (Ardouin, 2003, p. 23). Nous pouvons repérer trois grands types de métiers que vise ce diplôme : responsable de formation, responsable de formation en organisme de formation et formateur consultant. 


\subsection{Un dispositif de formation hybride avec douze jours de modalité en présentiel}

Le livret pédagogique précise que le processus de formation est pensé dans une logique progressive qu’implique « un continuum réflexif et rédactionnel ", lié à la validation d'unités d’enseignements construites en vue du développement d'une professionnalité d'ingénieur en formation. Il s'appuie sur une optimisation des interactions sociales, que ce soit : 1) avec des professionnels chevronnés (tuteurs en entreprise, intervenants praticiens de l'université), 2) les enseignants (qui ont la charge en particulier d'encadrer la réalisation des mémoires de recherches action), ou bien encore, 3) les pairs (lors des regroupements, des séminaires virtuels, des forums ou des regroupements) (cf. fig. 1).

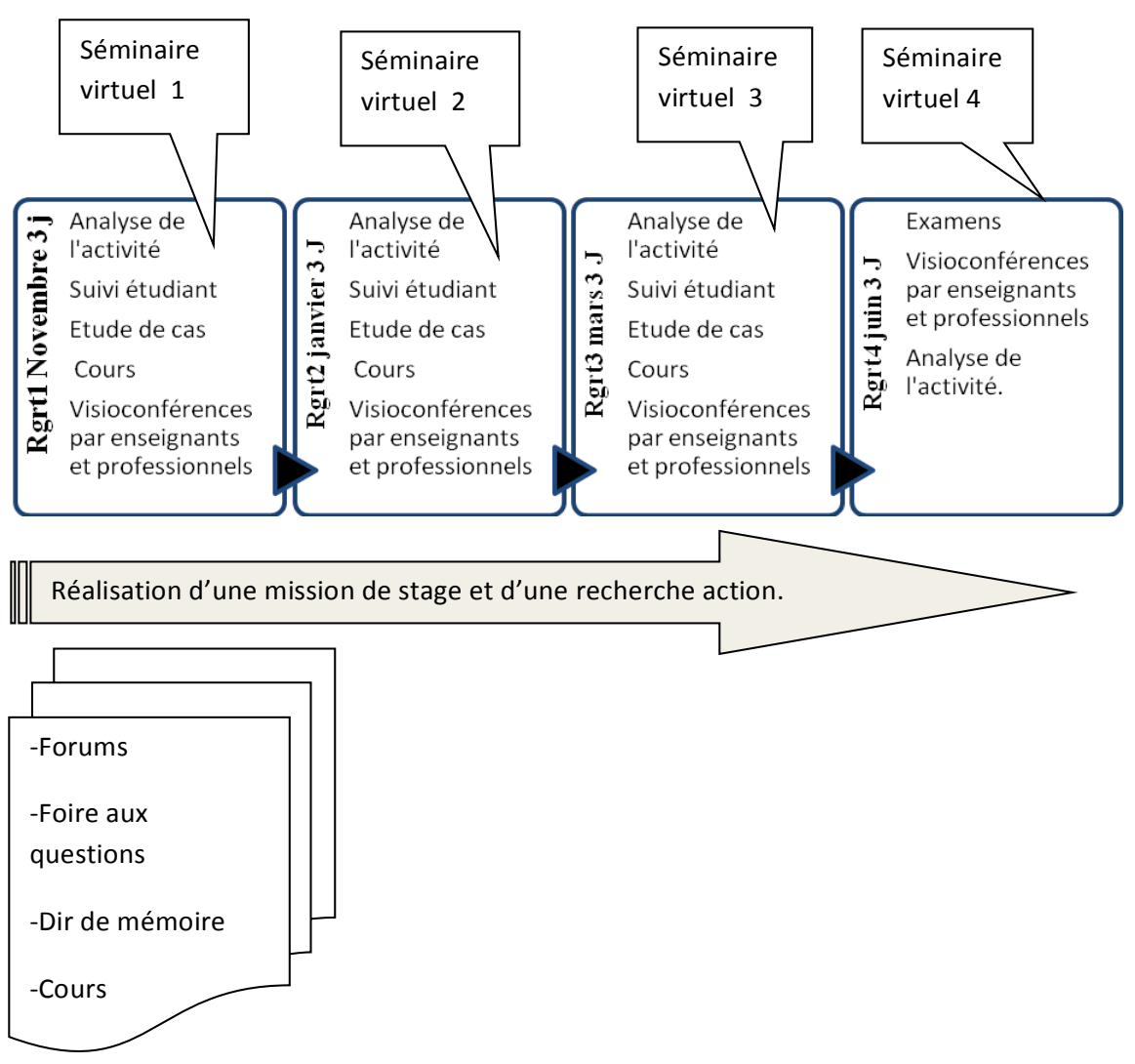

Figure 1 : Le dispositif de formation du master 2 Métiers de la formation de Rouen

Ces interactions doivent favoriser la distanciation de létudiant sur son action afin d’assurer la pensée réflexive, mais aussi afin de favoriser sa (trans)formation en encourageant le questionnement qui conduit à des prises de conscience, à donner du sens, soit à développer "l'ingenium " qui aborde la question du praticien non pas à partir de la question de ses connaissances, mais plutôt du savoir incorporé dans l'agir professionnel. De cette façon, peut surgir l’apprentissage, « dès lors qu’y émerge un conflit cognitif et que la régulation de ce conflit aboutit à l'ajustement et à la transformation des connaissances préalables mobilisées» (Bourgeois, 2009, p. 46). Cette distanciation qui s'inscrit ici dans la proximité (Triby, 2007) entre espace de réflexion et espace de l'action, nécessite de repenser l'espace de l’action et de définir au mieux l'objet de l'appropriation ou réappropriation sociale, soit de repenser également ses pratiques habituelles en terme de conditions de l'action. Bref de porter un nouveau regard sur des situations familières ou de les interroger dans une autre perspective, comme celle du développement d'une nouvelle professionnalité et/ou identité professionnelle.

\section{Méthodologie, analyse et présentations des résultats}

\subsection{Problématique et proposition d'hypothèses}

À l'expérience du stage s'ajoute celle de la formation à distance. Nous concevons cette situation particulière comme également potentiellement créatrice d'expériences vécues. Pour cela, les étudiants sont accompagnés individuellement dans un va et vient permanent entre leur mission et leur mémoire, dans des allers retours entre théorie et pratique ; entre réflexion et action. Ils sont également accompagnés dans leur 
découverte d'un dispositif hybride et dans cette nouvelle manière d'apprendre ou de construire leur savoir.

Mais ils sont également, dans le cadre de la formation professionnelle continue, des adultes en formation. C'est le rapport de l'adulte à ses activités qui le différencie de l’enfant ou de l’adolescent. Ainsi, selon Lesne (1984, p. 30), le fait que l'adulte «fait d’abord face à ses conditions d'existence (par le travail salarié ou non) et aux contraintes que celles-ci lui imposent ». La formation est par conséquent, selon ce dernier, caractérisée comme étant une activité seconde, mais qu’en est-il d'un adulte en stage?

Pour les étudiants de la formation professionnelle continue, il s’agit d’engager une remise en cause de leurs acquis précédemment éprouvés, qui peuvent être validés ou réfutés par l'expérimentation et la confrontation aux réalités de terrain. Est alors en œuvre ce que Piaget définissait comme le passage de l'assimilation à l'accommodation ; évolution nécessaire, induite et négociée qui entraîne un remaniement profond de ce qui constituait auparavant ses connaissances, soit la traversée d’une période de déséquilibre, qu'il est nécessaire d’accompagner, sous peine de le rendre hermétique à de nouveaux savoirs. C'est pourquoi, nous retiendrons la problématique suivante : Quels contenus des lettres de mission pour quelles démarches et activités de stage, pour des étudiants inscrits en formation professionnelle continue et suivant leurs études à distance? H1 : La conception des lettres de mission visent la réalisation de démarches d'ingénierie de formation, tel que cela est présenté dans le référentiel de formation du master, (Ardouin, 2003, p. 35), (cf. tableau. 1).

Tableau 1

La démarche d'ingénierie de formation

\begin{tabular}{|c|c|}
\hline Phases & Étapes \\
\hline Investigations & Analyser \\
\hline \multirow{2}{*}{ Mise en œuvre } & Concevoir \\
\cline { 2 - 2 } & Réaliser \\
\cline { 2 - 2 } & Concevoir \\
\hline
\end{tabular}

H2 : La conception des lettres de mission montre une grande diversité dans les démarches ou activités à mettre en œuvre. En cela, elles témoignent des composantes et de lévolution du travail dans le domaine de la formation.

\subsection{Une méthodologie de l'analyse des lettres de missions}

Nous portons une attention particulière à l'analyse de leurs composantes lexicales, aux éléments peu élaborés ou manquants, mais aussi aux dimensions individuelles ou collectives qui s'y trouvent exprimées. Nous avons fait le choix de retenir pour notre analyse $50 \%$ des lettres de mission (soit trente-trois) rédigées pour l’année 2012-2013, selon une logique de répartition proportionnelle aux effectifs étudiants de chaque site de regroupement; soit (dix-neuf de Rouen (dont quatre proviennent de : Tahiti, Mayotte, La Réunion, Bulgarie); cinq de Poitiers ; cinq de Fort-de-France ; trois du Burkina-Faso (Ouagadougou) ; un du Cameroun (Yaoundé)).

\subsubsection{Les lettres de mission: un outil d'ajustement et de régulation entre les différents acteurs de la formation}

Comme l'indique Bénédicte Goussault (2013), " un stage implique au moins trois partenaires, trois acteurs en interaction et avec des stratégies, des objectifs et des intérêts différents ». En tant quoutil de dialogue et d’ajustement, lécriture des lettres de missions peut être longue et comporte fréquemment trois grandes étapes. La première est à l'initiative de létudiant, elle consiste en la remise de la première version, soit le plus fréquemment, en la définition d'une commande et de ses conditions de réalisation. Il s’agit en général de l'expression d'un besoin émanant du lieu de mission, soit exprimé par l'employeur ou l'un de ses clients. S’ouvre alors le deuxième temps qui est celui du dialogue et de la concertation, dans le sens où cést aussi celui où l'université exprime elle aussi ses contraintes et ses souhaits, au regard de ce quelle connaît du contexte particulier de létudiant. Dans tous les cas, sera anticipée la faisabilité de cette commande par un étudiant, soit par une personne non spécialiste de l'action à réaliser (plan de formation, référentiel, audit, médiatisation, etc.), en lien avec les questions de pertinence et cohérence, au regard du contenu de formation. Létudiant se fera alors le messager de points de précisions à apporter ou des demandes de révisions, tout en s'assurant bien du sens de ces demandes. S'en suivra une autre proposition du commanditaire qui sera remaniée si nécessaire jusqu'à ce que l'ensemble des partenaires tombent d’accord et en valident le contenu.

Mais la lettre de mission peut également définir le contenu d'une auto-commande, ce qui correspond aux salariés qui ne bénéficient pas de tuteur interne, comme les salariés des très petits organismes de formation, les étudiants qui vivent à l'étranger (sites de l'AUF) ou quelques Français installés à létranger, dont l'environnement sociétal présente encore très peu de pratiques d'ingénierie en formation, ou bien encore aux étudiants ayant une activité d'indépendants (formateurs consultants indépendants). Dans ce cas, plus complexe à accompagner, létudiant se donnera à lui-même une mission, en questionnant ses besoins en lien avec son projet professionnel ; le but étant de parvenir à obtenir de l'université, l'apport d'un transfert de savoir-faire d'expérience ou d'expertise. Ici seront à clarifier tout autant, le besoin de formation de l'étudiant que la faisabilité du transfert attendu qui en résulte.

Comme outil d’ajustement entre des perceptions du travail différentes, de régulation entre des besoins parfois contradictoires, son écriture 
et sa validation s’appuie sur la capacité de létudiant à se faire médiateur et à s’assurer par son intermédiaire, que des échanges ont bien lieu jusquà l'obtention d'un accord. La lettre de mission manifeste alors une forme de contractualisation, entre la structure d'accueil de la mission, létudiant et le responsable pédagogique du diplôme, qui tous trois la signent. Une fois fait, le responsable pédagogique peut alors transmettre à létudiant un « accord pédagogique », retraçant les apprentissages visés et sur lesquels portera lévaluation de la mission.

\subsubsection{Des lettres de mission : outil de formalisation des dimensions pédagogiques}

L'écriture de la lettre de mission part d'une demande de la responsable pédagogique qui fournit à cette occasion une notice précisant les éléments attendus : 1) contenu ciblé de la mission ; 2) contexte et enjeux de la mission ; 3) contenu de la mission ; 4) modalités d'implication dans la mission ; 5) interlocuteurs internes et externes ; 6) formes de restitution ; 7) méthodologie envisagée ; 8) clauses de confidentialité ; 9) le planning de travail ; 10) les modalités de présence dans le lieu de mission (décharge, travail sur plusieurs lieux, approches comparées). Notre analyse des lettres de mission porte en particulier sur le contenu des points 1), 2), 3). Ces lettres doivent répondre aux attendus du diplôme, soit questionner les activités d’ingénierie de formation engagées durant la mission.

Ces activités sont décrites dans le livret pédagogique selon quatre grandes familles :

1. Analyser l'environnement, le contexte, la situation.

2. Concevoir, mobiliser et/ou élaborer des dispositifs de formation.

3. Animer, négocier et piloter.

4. Évaluer et auditer la formation (dispositifs, organisations, actions, ...).

Cest la présence de ces activités dans les missions que nous nous proposons d’interroger (cf. tableau 2).

Tableau 2

Grille d'analyse des lettres de missions

\begin{tabular}{|c|c|c|c|c|c|c|c|c|c|}
\hline 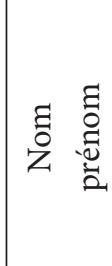 & 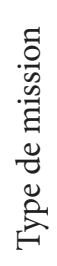 & 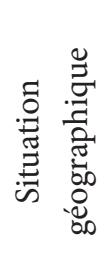 & 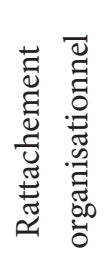 & 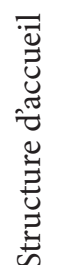 & 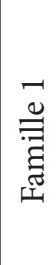 & 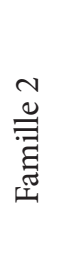 & 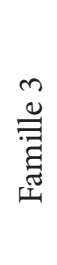 & 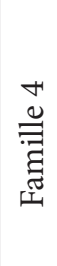 & 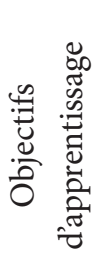 \\
\hline
\end{tabular}

\subsection{Présentations des résultats de l'analyse}

\subsubsection{Des lieux de stages appartenant prioritairement au service public de formation d'adultes}

Nous avons tout d'abord analysé les caractéristiques des lieux de stages. Pour des raisons d’anonymat, nous ne présentons pas ici lintégralité de nos résultats. Toutefois, les lieux d'accueil des stagiaires sont avant tout des structures publiques (onze établissements publics comme CCI, université, hôpital, Ministère, etc, ...) et neuf organismes de formation publics (GRETA 3, IFSI 3, CFA 1, IRTS 1, CIBC 1). Viennent ensuite les associations (5), et à parts égales, des grandes entreprises (2), des établissements privés (2), des organismes de formation privés (2) et enfin une grande école et un opérateur collecteur agréé (OPCA).

\subsubsection{Des contextes et enjeux des missions qui illustrent l'évolution du travail dans les métiers de la formation}

Il ne s'agit pas d'un stage d'observation mais véritablement de la mise en œuvre d'une démarche d'ingénierie ou de conseil en formation, laquelle s'accompagne d'une production : analyse de besoins, plan de formation, diagnostic socio organisationnel, conception d'un cahier des charges, construction d'un dispositif, évaluation d'action de formation ou audit de dispositif, etc., Létudiant construit son intervention à partir d'hypothèses de travail en vue d'une réalisation effective.

Nous pouvons classer les contextes et enjeux des missions selon quatre logiques : économiques; validation, valorisation et reconnaissance ; développement et diagnostic. Les lettres de mission donnent donc à voir le travail dans les métiers de la formation et ses évolutions. L’analyse des contextes illustre des missions mettant en évidence la nécessité d’une adaptation des organisations à différentes logiques, tant internes qu'externes.

1. Des logiques économiques, nous retenons en particulier les appels d’offres qui ont été introduits dans les processus d'achat du domaine de la formation. L'analyse lexicale fait ici ressortir des besoins en termes d'adaptation, comme : "moderniser le service public ", "trouver de nouvelles ressources financières ", ou bien encore "répondre à une nouvelle demande sociale ». Les enjeux déclarés sont stratégiques, il s'agit de préserver l'activité des organisations et de tout mettre en œuvre pour quelles puissent perdurer.

2. Des logiques de validation, valorisation et reconnaissance, sont également à mettre en relation avec les transformations du travail. Les besoins sont exprimés en matière de "professionnalisation des salariés ou des dispositifs »; ils peuvent également lêtre en termes de 
" réingénierie des référentiels » ou bien encore de " certification qualité » des organisations de travail ou de leurs salariés. Ici les enjeux déclarés sont la nécessité de démontrer un professionnalisme afin de se démarquer de la concurrence.

3. Des logiques de développement, nous retenons une vision stratégique interne des lieux d’accueil des missions. Les développements recherchés sont ceux de : «l’offre de formation », « des compétences », « du personnel », du capital humain ». Afin de pouvoir atteindre chacun de ces objectifs, des activités nouvelles sont créées : " offre de formation ", " dispositif ", "système dévaluation ", "plan de formation ", " structure », " accompagnement personnalisé ». Ici se jouent des questions stratégiques pour l’avenir des organisations concernées.

4. Enfin des logiques diagnostics sont associées à des activités de conseil en formation. Après avoir mis en œuvre une démarche d'audit, les étudiants devront dans ce cas, mener à bien des mesures d'impact, ou des plans d'amélioration. Ici sont exprimés des enjeux liés à la souffrance au travail, tel que "burn out ", « épuisement professionnel », ou des enjeux concernant des modalités de recrutement des personnels, afin de se démarquer de la concurrence. Les enjeux déclarés sont complexes et nécessitent une réelle capacité d’analyse et d'investigation, tels que " trouver un remède », ou « se donner un avantage », ainsi observe-ton des activités mettent en évidence la capacité réflexive et d’anticipation des étudiants.

Ces différentes logiques illustrent différentes facettes de l'ingénierie de la formation, mais surtout le fait qu’une démarche engagée nécessite de coordonner un grand nombre d'acteurs, individuels ou institutionnels. Ainsi, alors que l'on parle de plus en plus de "système formation ", l'activité du stagiaire est obligatoirement à questionner tant du point de vue individuel que collectif.

\subsubsection{Quelle(s) activité(s) engagée(s) pour quels contenus de stage?}

Reprenant le tableau 1 et la figure 2 présentés ci-dessus, cette analyse est basée sur une lecture des contenus de stages. Nous obtenons les résultats suivants : l'analyse de l'environnement, du contexte, ou de la situation de formation (domaine d'activités 1) est mentionnée à 21 reprises. La conception ou l'élaboration de dispositifs de formation (domaine d’activités 2), est indiqué 25 fois. L’animation, la négociation ou le pilotage (domaine d’activités 3), est affirmé 11 fois. Enfin, lévaluation et l'audit (domaine d'activités 4) sont exprimés à 17 reprises.

Nous pensons que cette cartographie des activités est à questionner au regard du degré d’expérience des stagiaires. De cette façon, il s’agit ici de la formulation d’objectifs d’apprentissage. En cela, il nous paraît peu surprenant que ce soit les activités de mise en œuvre (domaines d'activités 3 et 4) qui soient les moins représentées, alors que celles d'investigations (qui peuvent supposer une première expérience) (domaines d’activités 1 et 2) sont plus fréquemment mentionnées. Par ailleurs, les activités des domaines ( 3 et 4 ), sont celles que l'on acquiert avec l'expérience et constituent des domaines d'expertises (négociation, coordination, évaluation, audit).

Il s'en suit des combinaisons d'activités très variables d'un étudiant à un autre, parmi ces quatre grands domaines d'activités. Nous avons alors cherché à mesurer quelle était en moyenne, l’adéquation de ces missions au référentiel de formation du master. Pour cela, nous avons pour chaque étudiant, accordé un point par grande famille abordée. Nous obtenons de cette façon une moyenne de 2,1. Lanalyse des lettres de missions montre ainsi que la formation expérientielle d’un étudiant sur deux, permet d’explorer la moitié du référentiel de formation. Au final, seuls deux d’entre eux ont réalisé la démarche d’ingénierie enseignée dans sa totalité.

\subsubsection{Retour sur les hypothèses}

Notre première hypothèse était que les lettres de mission visent la réalisation de démarches d'ingénierie, telles que présentées dans le référentiel de formation du master. Nous venons de l'illustrer, nous devons l'infirmer, car en effet, leurs contenus correspondent davantage à l'intitulé du diplôme "métiers de la formation » et ouvrent sur un large éventail de combinaisons d'activités. De cette analyse des lettres de mission se dégage alors des représentations de l'ingénierie de formation dans le domaine professionnel, en tant que domaine d’activité introduit récemment dans le domaine de la formation (années quatre-vingt), l'ingénierie semble parfois encore méconnue et peu développée. "L'ingénierie apparaît ici comme un ensemble de techniques fondés sur une connaissance de la nature et des dynamiques de lacte de formation ", ces propos de Lesne (1990), correspondent bien à ce que nous avons pu observer. Nous devons également interroger la temporalité de l'acquisition d'une démarche d'ingénierie, soit l’apprentissage qui consiste dans la mise en œuvre de quatre grands domaines d'activités. Ici, la durée de la mission correspond à celle d'un stage long (450 heures), mais nous semble insuffisante sans une réelle connaissance préalable de l'un ou l'autre de ces grands domaines.

Notre deuxième hypothèse qui supposait que la conception des lettres de mission montrait une grande diversité dans les démarches ou activités et qu’elle témoignait des composantes et de l'évolution du travail dans le domaine de la formation est validée. Nous retenons de cette analyse une grande diversité des activités citées, mais aussi que les enjeux déclarés mettent en évidence, des politiques, des stratégies ou des conduites de changement qui impactent les organisations et le domaine de la formation en particulier. Nous retenons que cette analyse des lettres de mission ne peut être construite quà partir d'une définition préalable pour chaque étudiant d'objectifs d'apprentissage au travers d'une mission négociée. Ces objectifs propres à chaque étudiant ont été introduits dans les lettres de mission pour l’année 2013- 2014, mais n’apparaissaient pas dans celles, antérieures, que nous avons analysées. Elles illustrent également les éléments de la professionnalité du domaine de la formation, tant du point de vue du vocabulaire, que des éléments majeurs qui structurent l'ingénierie (dispositif, référentiel, appels d’offres, évaluation, etc.,). 


\section{Perspectives}

Lanalyse de l'activité n'est pas dans ce dispositif une modalité de formation, mais nous permet de questionner la conception du dispositif de formation, dans lequel les interactions sont indispensables au développement des apprentissages. En cela, nous retiendrons la définition d'un dispositif hybride comme étant : «Une instance, un lieu social d'interaction et de coopération possédant ses intentions, son fonctionnement matériel et symbolique enfin, ses modes d'interactions propres » (Peraya 1999, p. 153).

Le dispositif étudié favorise le tutorat par les pairs et le partage d'expériences. Il questionne l'expérience formative et le degré de distance nécessaire aux transactions entre l'apprenant et son environnement, Dewey (1938). Cette distance qui n'existe pas du point de vue de l'action rejoint en cela les travaux de Schön (1983/1994), pour lequel il s'agit de distinguer « la réflexion en cours d'action » et la « réflexion sur l'action ». Le travail de réflexivité comme outil et comme attitude devient alors indispensable pour ces stagiaires, en tant qu'aptitude humaine à se percevoir, soit " à reconsidérer, repenser, reconstruire mentalement ses expériences et ses actions d'une manière réfléchie » (Guillaumin, 2009, p. 169).

Pour ce faire, l'étudiant n'est pas seul, mais entourés d'une multitude d'acteurs : tuteur entreprise, pairs, référents de sites, intervenants professionnels, directeur de mémoire, mais il doit pouvoir répondre à une commande qui a été réellement négociée, qui permettra des apprentissages, au sens de l'expérience vécue. La non délimitation de l'espace de formation par rapport à celui du travail et/ou de la vie personnelle, n'est pas à interroger uniquement du point de vue pédagogique, elle doit être questionnée du point de vue axiologique qui concerne avant tout les finalités visées par la formation. S’agit-il de définir le contenu d'un stage tremplin, soit d'un stage qui ouvrira sur une autre fonction ultérieure ou une réorientation professionnelle ? S'agit-il d'un stage de perfectionnement facilitant la reconnaissance de compétences précédemment éprouvées ? S’agit-t-il d’un stage de mise en situation professionnelle dans la découverte d'une nouvelle fonction? Etc.

Cette activité pédagogique peu classique à l'université, que ce soit par l'utilisation des technologies ou de la forme d'interventions typiques de la formation des adultes, nécessite de repenser les formes d’accompagnement des nouveaux publics de l’université. Ici la faisabilité du suivi de stage est interrogée, pour des promotions d'environ 70 étudiants, répartis dans le monde. La lettre de mission semble alors être un outil de cadrage, d'ajustement et de régulation, mais ne peut se substituer à un véritable suivi des stagiaires dans leurs apprentissages. Nous interrogerons donc les effets produits par l'écriture de ces lettres et leur traduction en termes d'action. Ce travail d'évaluation des apprentissages réalisés pourra faire l’objet d'autres recherches.

La lettre de mission exprime donc à la fois les évolutions du travail (Labbé, Champy-Remoussenard, 2013), mais aussi la transformation progressive des relations éducation/formation/travail. Face aux injonctions à la professionnalisation des formations (Wittorski, 2012) au sein d'un système éducatif sans cesse interpellé quant à son adaptabilité au monde du travail, il nous semble que le dispositif présenté en est une belle tentative.

\section{Références bibliographiques}

Aballéa, F. (1992). Sur la notion de professionnalité. Recherche sociale, (124), p. 39-39.

Albarello, L., Barbier J-M., Bourgeois E. \& Durand M. (2013). Expérience, activité, apprentissage. Paris : PUF.

Ardoino, J. (1990). Les postures (ou impostures) respectives du chercheur, de l'expert et du consultant. Consulté le 15 janvier 2013 sur http:// www.arianesud.com/bibliotheque/aa auteurs/ardoino/ardoino postures et impostures 19902.

Ardouin, T. (2003). Ingénierie de formation pour l'entreprise. Analyser, concevoir, réaliser, évaluer. Paris : Dunod.

Ardouin, T. (2007). Ce qui compte dans les formations à distance, c'est la présence ! Le cas du master ICF. Dans J. Wallet (dir.). Le campus numérique FORSE : analyses et témoignages (83-90). Rouen : Presses des universités de Rouen et du Havre.

Barbier, J.-M. (1992). La recherche de nouvelles formes de formation par et dans les situations de travail. Éducation Permanente, (112).

Barbier, J.-M. (2013). Expérience, apprentissage, éducation. Dans L. Albarello, J.-M. Barbier, E. Bourgeois \& M. Durand, Expérience, activité, apprentissage (65-85). Paris : PUF.

Balleux, A. (2000). Évolution de la notion d'apprentissage expérientiel en éducation des adultes : vingt-cinq ans de recherche. Revue des sciences de l'éducation, 26(2), p. 263-286.

Bourgeois, E. \& Nizet J. (1997). Apprentissage et formation des adultes. Paris : PUF.

Bourgeois, E. (2009).Le s dispositifs d'apprentissage en formation. Dans J.-M. Barbier, E. Bourgeois, G. Chapelle, \& J.-C. Ruano-Borbalan (dir.), Encyclopédie de la formation. Paris : PUF, p. 507-536.

Bourgeois E. \& Durand, M. (2012). Apprendre au travail. Paris : PUF.

Boutinet, J-P. (1990). Anthropologie de projet. Paris : PUF.

Caspar, P. (2011). La formation des adultes hier, aujourd'hui, demain. Eyrolles : Éditions d'Organisation.

Champy-Remoussenard, P. (2005). Les théories de l'activité entre travail et formation. Savoirs, (8), p. 9-50.

Depover, C. \& Marchand, L. (2002). E-learning et formation des adultes en contexte professionnel. Bruxelles : de Boeck.

Dewey, J. (1938). Expérience and Education. Kappa : Delta Pi.

Dewey, J. (2006). Logique, la théorie de l'enquête. Paris : PUF. 
Di Meo, G. (2001). Les nouveaux territoires du social. APSAM.

Goussalt, B. (2013). Un stage, trois acteurs. V. de Briant, \& D. Glaymann (dir.). Le stage. Formation ou exploitation ?(71-79). Rennes : Presses Universitaires de Rennes.

Guillaumin, C. (2009). Lobservation réflexive en formation. Dans C. Guillaumin, S. Pesce \& N. Denoyel (dir.), Pratiques réflexives en formation Ingéniosité et ingénieries émergentes(155-181). Paris : L’Harmattan.

Houssaye, J. (2012). Former à la pédagogie : oui, mais comment? Penser léducation, (32), p. 5-14.

Kolb, D. A. (1984). Experiential Learning. Experience as the Source of Learning and Development. Englewood Cliffs, NJ : Prentice-Hall.

Labbé, S. \& Champy-Remoussenard, P. (2013). Le travail en évolution. Les Dossiers des sciences de léducation, (30).

Leplat, J. (1997). Regards sur l'activité en situation de travail. Paris : PUF.

Leplat, J. (2000). L’environnement de l’action en situation de travail. Actes du séminaire de recherche sur la formation du CNA, Lanalyse de la singularité de laction (107-132). Paris : PUF.

Lesne, M. (1984). Lire les pratiques de formation d'adulte. Paris : Edilig.

Lesne, M. \& Minvielle Y. (1990). Socialisation et formation. Paris : Païdea.

Madelrieux, S. (2010). Le pragmatisme et les variétés de l’expérience. Dans L. Perreau (dir.), Leexpérience. Paris : Vrin.

Mayen, P. (2009). Expérience et formation des adultes. Dans J.-M. Barbier, E. Bourgeois, G. Chapelle \& J.-C. Ruano-Borbalan (dir.). Encyclopédie de la formation, p. 763-780.

Mezzena, S. (2011). L'expérience du stagiaire en travail social : le point de vue situé de l’activité. Pensée plurielle, (26), p. 37-51.

Paquelin, D., Audran, J., Chopelin, H., Hryshchuk, S. et Simonian, S. (2006). Campus numériques et innovation pédagogique : L’hypothèse de la territorialisation. Distances et savoirs, 3(4), p. 365-395.

Pastré, P. (2013). Le travail de lexpérience. Dans L. Albarello, J.-M. Barbier, E. Bourgeois \& M. Durand, Expérience, activité, apprentissage. Paris : PUF.

Peraya, D. (1999). Médiation et médiatisation : le campus virtuel. Vers les campus virtuels . Hermès, (25), p. 153-167.

Perez-Roux T. (2012). La professionnalité enseignante. Modalités de construction en formation. Rennes : PUR.

Schön, D. A. (1994). Le praticien réflexif. À la recherche du savoir caché dans l'agir professionnel. Montréal : Éditions Logiques.

Triby, E. (2007). La distance en formation : une proximité tenace ", dans E. Triby \& E. Heilmann (dir.). À distance. Apprendre, travailler, communiquer. Strasbourg : Presses universitaires de Strasbourg.

Wittorski R. (2012). La professionnalisation de l’offre universitaire : Quelles spécificités ", dans Revue internationale de pédagogie de lenseignement supérieur, Consulté le 20 avril 2012 sur : http://ripes.revues.org/580 